\title{
PULSATING FRONT SPEED-UP AND QUENCHING OF REACTION BY FAST ADVECTION
}

\author{
ANDREJ ZLATOŠ
}

\begin{abstract}
We consider reaction-diffusion equations with combustion-type non-linearities in two dimensions and study speed-up of their pulsating fronts by general periodic incompressible flows with a cellular structure. We show that the occurence of front speed-up in the sense $\lim _{A \rightarrow \infty} c_{*}(A)=\infty$, with $A$ the amplitude of the flow and $c_{*}(A)$ the (minimal) front speed, only depends on the geometry of the flow and not on the reaction function. In particular, front speed-up occurs for KPP reactions if and only if it does for ignition reactions. We provide a sharp characterization of the periodic symmetric flows which achieve this speed-up and also show that these are precisely those which, when scaled properly, are able to quench any ignition reaction.
\end{abstract}

\section{INTRODUCTION AND EXAMPLES}

In this paper we study the effects of strong incompressible advection on combustion. We consider the reaction-advection-diffusion equation

$$
T_{t}+A u(x) \cdot \nabla T=\Delta T+f(T), \quad T(0, x)=T_{0}(x) \in[0,1]
$$

on $D \equiv \mathbb{R} \times \mathbb{T}^{d-1}$, with $u$ a prescribed flow profile and $A \gg 1$ its amplitude. Here $T(t, x) \in$ $[0,1]$ is the normalized temperature of a premixed combustible gas and $f$ is the burning rate.

We assume that $u \in C^{1, \varepsilon}(D)$ is a periodic incompressible (i.e., $\nabla \cdot u \equiv 0$ ) vector field which is symmetric across the hyperplane $x_{1}=0$. That is, $u(R x)=R u(x)$ where $R\left(x_{1}, \ldots, x_{d}\right)=$ $\left(-x_{1}, x_{2}, x_{3}, \ldots, x_{d}\right)$ is the reflection across $x_{1}=0$. If the period of $u$ in $x_{1}$ is $p$, then this implies that $u$ is symmetric across each hyperplane $x_{1}=k p, k \in \mathbb{Z}$. Hence $u$ is a periodic symmetric flow of cellular type ( since $u_{1}(x)=0$ when $x_{1} \in p \mathbb{Z}$ ) with $[0, p] \times \mathbb{T}^{d-1}$ a cell of periodicity.

The reaction function $f \in C^{1, \varepsilon}([0,1])$ is of combustion type. That is, there is $\theta_{0} \in[0,1)$ such that $f(s)=0$ for $s \in\left[0, \theta_{0}\right] \cup\{1\}$ and $f(s)>0$ for $s \in\left(\theta_{0}, 1\right)$, and $f$ is non-increasing on $(1-\varepsilon, 1)$ for some $\varepsilon>0$. This includes the ignition reaction term with $\theta_{0}>0$ and positive reaction term with $\theta_{0}=0$. In the latter case we single out the Kolmogorov-PetrovskiiPiskunov (KPP) reaction [13] with $0<f(s) \leq s f^{\prime}(0)$ for all $s \in(0,1)$.

We will be interested in two effects of the strong flow $A u$ on combustion: pulsating front speed enhancement and quenching of reaction. This problem has recently seen a flurry of activity - see [1, 3, 5, 6, 7, 8, 9, 10, 11, 12, 15, 18, 22, 23, 24. A pulsating front is a solution of (1.1) of the form $T(t, x)=U\left(x_{1}-c t, x\right)$, with $c$ the front speed and $U(s, x)$ periodic in $x_{1}$ (with period $p$ ) such that

$$
\lim _{s \rightarrow-\infty} U(s, x)=1 \quad \text { and } \quad \lim _{s \rightarrow+\infty} U(s, x)=0,
$$


uniformly in $x$. It is well known [4] that in the case of positive reaction there is $c_{*}(A)$, called the minimal pulsating front speed, such that pulsating fronts exist precisely for speeds $c \geq c_{*}(A)$. In the ignition reaction case the front speed is unique and we again denote it $c_{*}(A)$. In the present paper we will be interested in the enhancement of this (minimal) front speed by strong flows.

We say that the flow $A u$ quenches (extinguishes) the initial "flame" $T_{0}$ if the solution of (1.1) satisfies $\|T(t, \cdot)\|_{\infty} \rightarrow 0$ as $t \rightarrow \infty$. Here one usually considers compactly supported initial data. The flow profile $u$ is said to be quenching for the reaction $f$ if for any compactly supported initial datum $T_{0}$ there is an amplitude $A_{0}$ such that $T_{0}$ is quenched by the flow $A u$ whenever $A \geq A_{0}$. We note that quenching never happens for KPP reactions - the solutions of (1.1) for compactly supported non-zero $T_{0}$ always propagate and the speed of their spreading equals $c_{*}(A)$ [4, 20].

In this paper we characterize those periodic symmetric incompressible flows in two dimensions which achieve speed-up of fronts and, if scaled properly, quenching of any ignition reaction. For $l>0$ we denote by $l \mathbb{T}$ the interval $[0, l]$ with its ends identified, and we let $u^{(l)}(x) \equiv u(x / l)$ be the scaled flow on $\mathbb{R} \times l \mathbb{T}$ (with cells of size $l p \times l$ ).

Theorem 1.1. Let $u$ be a $C^{1, \varepsilon}$ incompressible p-periodic flow on $D=\mathbb{R} \times \mathbb{T}$ which is symmetric across $x_{1}=0$, and let $f$ be any combustion-type reaction.

(i) If the equation

$$
u \cdot \nabla \psi=u_{1}
$$

on $p \mathbb{T} \times \mathbb{T}$ has a solution $\psi \in H^{1}(p \mathbb{T} \times \mathbb{T})$, then

$$
\limsup _{A \rightarrow \infty} c_{*}(A)<\infty
$$

and no $u^{(l)}$ is quenching for $f$.

(ii) If (1.2) has no $H^{1}(p \mathbb{T} \times \mathbb{T})$-solutions, then

$$
\lim _{A \rightarrow \infty} c_{*}(A)=\infty
$$

and if $f$ is of ignition type, then there is $l_{0} \in(0, \infty)$ such that the flow $u^{(l)}$ on $\mathbb{R} \times l \mathbb{T}$ is quenching for $f$ when $l<l_{0}$ and not quenching when $l>l_{0}$.

Remarks. 1. The proof shows that in (ii), $l_{0} \geq c\|f(s) / s\|_{\infty}^{-1 / 2}$ for some $u$-independent $c>0$. It can also be showed that the claim $l_{0}>0$ in (ii) extends to some positive reactions that are weak at low temperatures (more precisely, $f(s) \leq \alpha s^{\beta}$ for some $\alpha>0$ and $\beta>3-$ see Corollary 4.4), in particular, the Arrhenius reaction $f(s)=e^{-C / s}(1-s), C>0$. On the other hand, if $f(s) \geq \alpha s^{\beta}$ for some $\alpha>0, \beta<3$, and all small $s$, then $l_{0}=0$ for any $u[22]$.

2. We note that $l_{0}=\infty$ is impossible for cellular flows in two dimensions - see [23] which studies strongly quenching flows $u$, that is, quenching for any ignition reaction and any $l$.

3. Although we only consider periodic boundary conditions here, it is easy to see that Theorem 1.1 remains valid for (1.1) on $\mathbb{R} \times[0,1]$ with Neumann boundary conditions, provided $u_{2}(x)=0$ when $x_{2} \in\{0,1\}$. 
4. Although a part of our analysis - Sections 2 and 3 - is valid in any dimension, it remains an open quenstion whether Theorem 1.1 also extends beyond two dimensions.

Theorem 1.1 has the following corollary:

Corollary 1.2. Let $u$ be a $C^{1, \varepsilon}$ incompressible p-periodic flow on $D=\mathbb{R} \times \mathbb{T}$ which is symmetric across $x_{1}=0$. Then speed-up of pulsating fronts by $u$ in the sense of (1.4) occurs for ignition reactions if and only if it occurs for KPP reactions.

Remark. Although speed-up of KPP fronts has been studied extensively (see, e.g., 3, 5, 6, 10, 11, 15, 18, 24]), rigorous results on ignition front speed-up have so far been established only in two dimensions for percolating flows and special cellular flows [11] (see below).

It is not surprising that the flows which achieve speed-up of fronts are precisely those which quench large initial data. Fast fronts are long, the latter being due to short time-long distance mixing by the underlying flow. Such mixing yields quenching, although possibly only away from regions where the flow is relatively still (e.g., the centers of the cells in Figure 1 below). If these regions are sufficiently small, for instance when the flow is scaled, then reaction cannot survive inside them and global quenching follows. This relation of front speed to flow mixing properties also illuminates Corollary 1.2.

Note that the above assumptions on $u$ exclude the class of percolating flows (in particular, shear flows $\left.u(x)=\left(\alpha\left(x_{2}, \ldots, x_{d}\right), 0, \ldots, 0\right)\right)$ which possess streamlines connecting $x_{1}=-\infty$ and $x_{1}=+\infty$. In two dimensions, the conclusions of Theorem 1.1(ii) for these flows have been established in [6, 17, 11, 12, 18]. Moreover, results from [5, 24] can be used to prove linear pulsating front speed-up (namely, $\lim _{A \rightarrow \infty} c_{*}(A) / A>0$ ) by percolating flows in the presence of KPP reactions in any dimension.

As for cellular flows in two dimensions (the kind we consider here), the claims about the front speed $c_{*}(A)$ in Theorem 1.1 have been proved for KPP reactions in [18]. The special case of the flow $u(x)=\nabla^{\perp} H(x) \equiv\left(-H_{x_{2}}, H_{x_{1}}\right)$ with the stream function $H\left(x_{1}, x_{2}\right)=$ $\sin 2 \pi x_{1} \sin 2 \pi x_{2}$ has been addressed in [9, 11, 15], which proved (1.4) for any reaction and quenching by $u^{(l)}$ for small enough $l$ and ignition reactions. The streamlines of this flow are depicted in Figure 1.

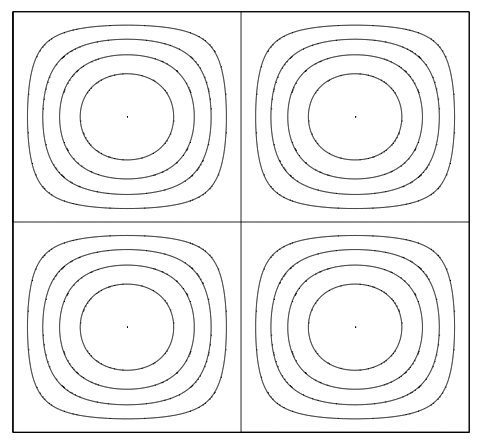

Figure 1. A cellular flow. 
We note that it is easy to show that (1.2) has no $H^{1}\left(\mathbb{T}^{2}\right)$-solutions in this case [18], and so one can recover these results from Theorem 1.1(ii). Our general method does not yield the more precise asymptotics $c_{*}(A) \sim A^{1 / 4}$ in the KPP case [15] and $A^{1 / 5} \lesssim c_{*}(A) \lesssim A^{1 / 4}$ in the ignition case [1] for this particular flow.

We conclude this introduction with two more examples of types of flows to which Theorem 1.1 applies.

Example 1.3. Checkerboard flows. Consider the cellular flow above vanishing in every other cell as depicted in Figure 2, thus forming a checkerboard-like pattern. This flow is both periodic (with period 2) and symmetric but it is not $C^{1, \varepsilon}$. Let us remedy this problem by letting the stream function be $H\left(x_{1}, x_{2}\right)=\left(\sin 2 \pi x_{1} \sin 2 \pi x_{2}\right)^{\alpha}$ with $\alpha>2$ in the cells where $u$ does not vanish. Again, (1.2) has no $H^{1}(2 \mathbb{T} \times \mathbb{T})$-solutions [18], and so Theorem 1.1(ii) speed-up of fronts and quenching by $u^{(l)}$ - holds. Moreover, the same conclusion is valid for other flows with this type of structure, even if the angle of contact of the "active" cells is $\pi$.

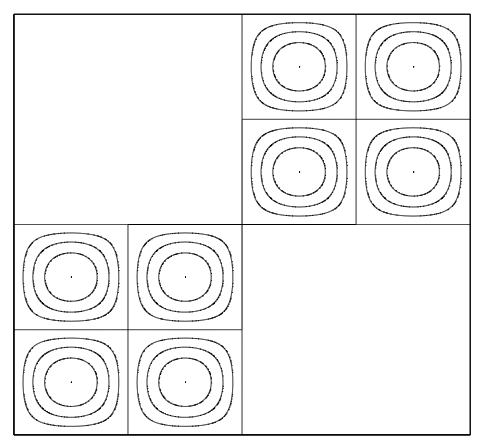

Figure 2. A checkerboard cellular flow.

Example 1.4. Flows with gaps. Consider again the cellular flow above but with a vertical "gap" of width $\delta>0$, in which the flow vanishes, inserted in place of each vertical segment $\{k\} \times \mathbb{T}, k \in \mathbb{Z}$, such as shown in Figure 3. We again need to alter the stream function as we did in the previous example in order to make the flow $C^{1, \varepsilon}$. This time it is easy to see that (1.2) has $H^{1}((1+\delta) \mathbb{T} \times \mathbb{T})$-solutions [18, and so Theorem 1.1(i) - no speed-up of fronts and no quenching by $u^{(l)}$ - holds in this case. The same conclusion is valid for other flows with similar structures of streamlines, even when the gaps are replaced by channels in which the flow moves "along" the channel only (see [18] for more details).

We also note that Sections 2 and 3 below yield the conclusions of Theorem 1.1(i) for cellular flows with gaps in any dimension (using that gaps force Lemma 2.2(ii) to hold).

The rest of the paper consists of Section 2 where we prove a few preliminary lemmas, and Sections 3 and 4 which contain the proof of Theorem 1.1 .

The author would like to thank Sasha Kiselev, Tom Kurtz, and Greg Lawler for useful discussions. Partial support by the NSF through the grant DMS-0632442 is also acknowledged. 


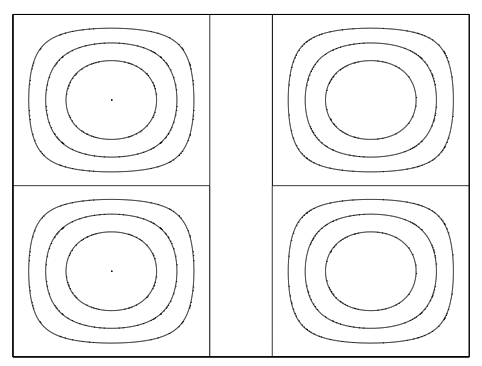

Figure 3. A cellular flow with gaps.

\section{Some Preliminaries}

In this and the next two sections we will assume the hypotheses of Theorem 1.1 with the period $p=1$ - the general case is handled identically. This implies that $u$ is symmetric across each hyperplane $x_{1}=k, k \in \mathbb{Z}$. The analysis in this section and the next applies to (1.1) on $D=\mathbb{R} \times \mathbb{T}^{d-1}$ for any $d \in \mathbb{N}$.

Let us consider the stochastic process $X_{t}^{A, x}$ starting at $x \in D$ and satisfying the stochastic differential equation

$$
d X_{t}^{A, x}=\sqrt{2} d B_{t}-A u\left(X_{t}^{A, x}\right) d t, \quad X_{0}^{A, x}=x,
$$

where $B_{t}$ is a normalized Brownian motion on $D$. We note that by Lemma 7.8 in [16], we have that if

$$
\phi_{t}+A u(x) \cdot \nabla \phi=\Delta \phi, \quad \phi(0, x)=\phi_{0}(x),
$$

then

$$
\phi(t, x)=\mathbb{E}\left(\phi_{0}\left(X_{t}^{A, x}\right)\right) .
$$

In particular, $\phi_{0}(x)=\chi_{[-L, L]}(x)$ gives

$$
\phi(t, x)=\mathbb{P}\left(\left|X_{t}^{A, x}\right| \leq L\right),
$$

where we define $|x| \equiv\left|x_{1}\right|$ for $x \in D$. Also notice that if $\phi_{0}=T_{0} \in[0,1]$, then by comparison theorems [19] for any $t, x$,

$$
0 \leq T_{0}(t, x) \leq e^{t\|f(s) / s\|_{\infty}} \phi(t, x) \leq e^{t\left\|f^{\prime}\right\|_{\infty}} \phi(t, x) .
$$

Lemma 2.1. (i) If $k \in \mathbb{Z}$ and $y_{1}=k$ then the distribution of $X_{t}^{A, y}$ is symmetric across the hyperplane $x_{1}=k$, that is,

$$
\mathbb{P}\left(X_{t}^{A, y} \in V\right)=\mathbb{P}\left(X_{t}^{A, y} \in R(V-(k, 0))+(k, 0)\right)
$$

for each $V \subseteq D$.

(ii) If $k \in \mathbb{Z}$ and $y_{1} \geq k$, then for any $I \subseteq \mathbb{R}^{+}$,

$$
\mathbb{P}\left(\left(X_{t}^{A, y}\right)_{1} \in k+I\right) \geq \mathbb{P}\left(\left(X_{t}^{A, y}\right)_{1} \in k-I\right) .
$$

When $y_{1} \leq k$, the inequality in (2.6) is reversed. 
(iii) If $L \in \mathbb{N}$, then

$$
\mathbb{P}\left(\left|X_{t}^{A, y}\right| \leq L\right) \leq\left\lceil\frac{\left|y_{1}\right|}{L}\right\rceil^{-1} .
$$

Proof. (i) and (ii) are obvious from the symmetry of $u$ across $x_{1}=k$ and from almost sure continuity of $X_{t}^{A, y}$ in $t$. To show (iii), it is sufficient to consider $y_{1}>L$. Applying (ii) with $k=j L$ for $j=1, \ldots,\left\lceil y_{1} / L\right\rceil-1$, we see that

$$
\mathbb{P}\left(\left(X_{t}^{A, y}\right)_{1} \in[-L, L]\right) \leq \mathbb{P}\left(\left(X_{t}^{A, y}\right)_{1} \in[(2 j-1) L,(2 j+1) L]\right) .
$$

The claim follows.

Next we prove the following key dichotomy.

Lemma 2.2. For any sequence $\left\{A_{n}\right\}_{n=1}^{\infty}$ one of the following holds.

(i) For any $t, \varepsilon>0$ and $L<\infty$ there are $x, n$ such that

$$
\mathbb{P}\left(\left|X_{t}^{A_{n}, x}-x\right| \leq L\right)<\varepsilon .
$$

(ii) For any $t, \varepsilon>0$ there is $L<\infty$ such that for any $x, n$,

$$
\mathbb{P}\left(\left|X_{t}^{A_{n}, x}-x\right| \leq L\right)>1-\varepsilon .
$$

Proof. Let us first assume that there is $t^{\prime}>0$ such that for any $\varepsilon^{\prime}>0$ and $L^{\prime}<\infty$ there are $x, n$ such that

$$
\mathbb{P}\left(\left|X_{t^{\prime}}^{A_{n}, x}-x\right| \leq L^{\prime}\right)<\varepsilon^{\prime} .
$$

Given any $\varepsilon>0, L \in \mathbb{N}$, let $m>2 / \varepsilon$ be an integer and let $x, n$ be as in (2.10) with $\varepsilon^{\prime}=1 / m$, $L^{\prime}=(2 m+1) L$. Notice that by periodicity of $u$ we can assume $\left|x_{1}\right| \leq 1$. For any $t \geq t^{\prime}$ we have

$$
\mathbb{P}\left(\left|X_{t}^{A_{n}, x}-x\right| \leq L\right) \leq \mathbb{P}\left(\left|X_{t}^{A_{n}, x}\right| \leq 2 L\right) \leq \mathbb{P}\left(\left|X_{t^{\prime}}^{A_{n}, x}\right| \leq 2 m L\right)+\sup _{|y| \geq 2 m L} \mathbb{P}\left(\left|X_{t-t^{\prime}}^{A_{n}, y}\right| \leq 2 L\right) .
$$

The first term is smaller than $\varepsilon^{\prime}<\varepsilon / 2$ by (2.10) and the second is at most $1 / m<\varepsilon / 2$ by (2.7). This yields (i) for $t \geq t^{\prime}$. On the other hand, if (i) does not hold for some $t \in\left(0, t^{\prime}\right)$, then there are $\varepsilon, L$ such that for all $x, n$,

$$
\mathbb{P}\left(\left|X_{t}^{A_{n}, x}-x\right| \leq L\right) \geq \varepsilon
$$

Choose $m \in \mathbb{N}$ so that $m t \geq t^{\prime}$. It follows that

$$
\mathbb{P}\left(\left|X_{m t}^{A_{n}, x}-x\right| \leq m L\right) \geq \varepsilon^{m}
$$

for all $x, n$. But this contradicts (i) for $m t$, which has just been proven. Therefore (i) holds for all $t>0$ under the hypothesis above.

Now assume the opposite case to the one above. Namely, that for each $t^{\prime}>0$ there are $\varepsilon^{\prime}>0$ and $L^{\prime}<\infty$ such that for all $x, n$,

$$
\mathbb{P}\left(\left|X_{t^{\prime}}^{A_{n}, x}-x\right| \leq L^{\prime}\right) \geq \varepsilon^{\prime} .
$$

We will show that then (ii) holds, thus finishing the proof. 
For each $t>0$ let

$$
\varepsilon_{0}(t) \equiv \sup _{L} \inf _{x, n} \mathbb{P}\left(\left|X_{t}^{A_{n}, x}-x\right| \leq L\right)>0
$$

Periodicity of $u$ guaranties that

$$
\varepsilon_{0}(t)=\sup _{L \in \mathbb{N}} \inf _{|x| \leq 1, n} \mathbb{P}\left(\left|X_{t}^{A_{n}, x}\right| \leq L\right)\left(\equiv \sup _{L \in \mathbb{N}} \varepsilon_{1}(t, L)\right) .
$$

Notice that $\varepsilon_{0}(t)$ is non-increasing. Indeed, for $L, m \in \mathbb{N}$ and $t \geq t^{\prime}$,

$$
\varepsilon_{1}(t, L) \leq \varepsilon_{1}\left(t^{\prime}, m L\right)+\frac{1}{m}
$$

by (2.7), and so $\varepsilon_{0}(t) \leq \varepsilon_{0}\left(t^{\prime}\right)+1 / m$ for any $m$.

We will now show that $\varepsilon_{0}(t)=1$ for all $t$. To this end assume $\varepsilon_{0}(t)<1$ for some $t$. Let $m$ be large (to be chosen later), and let $L$ be such that

$$
\varepsilon_{1}(t, L)>\varepsilon_{0}(t)-\frac{1}{m}
$$

Consider any $|x| \leq 1, n$ such that

$$
\mathbb{P}\left(\left|X_{t}^{A_{n}, x}\right| \leq(2 m+1) L\right) \leq \varepsilon_{0}(t)+\frac{1}{m} .
$$

Such $x, n$ do exists because of $\varepsilon_{0}(t) \geq \varepsilon_{1}(t,(2 m+1) L)$. Then the set of Brownian paths for which there is $t^{\prime} \in[0, t]$ such that $\left|X_{t-t^{\prime}}^{A_{n}, x}\right|=(m+1) L$ has measure at least $1-\varepsilon_{0}(t)-1 / m$. Since

$$
\begin{gathered}
\mathbb{P}\left(\left|X_{t}^{A_{n}, x}\right| \in[L,(2 m+1) L]|| X_{t-t^{\prime}}^{A_{n}, x} \mid=(m+1) L \text { for some } t^{\prime} \in[0, t]\right) \\
\geq \inf _{t^{\prime} \in[0, t]} \varepsilon_{1}\left(t^{\prime}, m L\right)>\varepsilon_{0}(t)-\frac{2}{m}
\end{gathered}
$$

by (2.12) and (2.13), this means

$$
\begin{aligned}
\mathbb{P}\left(\left|X_{t}^{A_{n}, x}\right| \leq(2 m+1) L\right) & =\mathbb{P}\left(\left|X_{t}^{A_{n}, x}\right| \leq L\right)+\mathbb{P}\left(\left|X_{t}^{A_{n}, x}\right| \in[L,(2 m+1) L]\right) \\
& \geq \varepsilon_{1}(t, L)+\left(1-\varepsilon_{0}(t)-\frac{1}{m}\right)\left(\varepsilon_{0}(t)-\frac{2}{m}\right) \\
& \geq\left(2-\varepsilon_{0}(t)-\frac{1}{m}\right)\left(\varepsilon_{0}(t)-\frac{2}{m}\right) .
\end{aligned}
$$

Since $\varepsilon_{0}(t)<1$, this is larger than $\varepsilon_{0}(t)+1 / m$ when $m$ is large enough. This, however, contradicts (2.14). Therefore we must have $\varepsilon_{0}(t)=1$ for all $t$, which is (ii).

We will also need the following result which is essentially from [8].

Lemma 2.3. For any $d \in \mathbb{N}$, there is $c>0$ such that for any Lipschitz incompressible flow $u$, any $A$, and any $t \geq 0$, the solution $\phi$ of $(2.2)$ on $\Omega \equiv[0,1] \times \mathbb{T}^{d-1}$ with Dirichlet boundary conditions on $\partial \Omega$ satisfies

$$
\|\phi(t, \cdot)\|_{\infty} \leq 2 e^{-c t}\left\|\phi_{0}\right\|_{\infty}
$$


Proof. The maximum principle implies that it is sufficient to show that there is $\tau>0$ such that

$$
\|\phi(\tau, \cdot)\|_{\infty} \leq \frac{1}{2}\left\|\phi_{0}\right\|_{\infty}
$$

uniformly in $u$ and $A$. For incompressible flows on $\mathbb{T}^{d}$ and mean-zero $\phi_{0}$ this follows from Lemma 5.6 in [8]. The proof extends without change to our case, the Dirichlet boundary condition replacing the mean-zero assumption when the Poincaré inequality is used.

\section{Proof of Theorem 1.1: PART I}

Let us now assume that $u$ and $f$ are as in Theorem 1.1 and $A_{n} \rightarrow \infty$ is such that Lemma 2.2 (ii) holds. We will then show that the minimal front speeds $c_{*}\left(A_{n}\right)$ are uniformly bounded and the flows $A_{n} u$ do not quench large enough compactly supported initial data $T_{0}$ for (1.1). The analysis in this section applies to $D=\mathbb{R} \times \mathbb{T}^{d-1}$ for any $d \in \mathbb{N}$.

Lemma 3.1. Consider the setting of Theorem 1.1 with $D=\mathbb{R} \times \mathbb{T}^{d-1}$, and let $A_{n} \rightarrow \infty$ be such that Lemma 2.2(ii) holds. Then $c_{*}\left(A_{n}\right)$ are uniformly bounded above.

Proof. Choose $L \in \mathbb{N}$ that satisfies Lemma 2.2(ii) for $t=1$ and $\varepsilon=\frac{1}{4}$. Let $x$ be such that $x_{1} \in \mathbb{Z}$ and consider $X_{t}^{A_{n}, x}$ from (2.1). Take $\tau_{0}=0$ and let $\tau_{j}$ be the first time such that $\left|X_{\tau_{j}}^{A_{n}, x}-X_{\tau_{j-1}}^{A_{n}, x}\right|=3 L$ (recall that $\left.|x|=\left|x_{1}\right|\right)$. We then have from (2.9) and (2.7),

$$
\mathbb{P}\left(\tau_{j}-\tau_{j-1} \leq 1\right) \leq \frac{1}{2}
$$

because $\frac{1}{3} p+(1-p) \geq \frac{3}{4}$ implies $p \leq \frac{1}{2}$. This means that for any large enough $C, t \in \mathbb{N}$,

$$
\begin{aligned}
\mathbb{P}\left(\left|X_{t}^{A_{n}, x}-x\right| \geq 3 L C t\right) \leq \mathbb{P}\left(\tau_{C t} \leq t\right) & \leq \sum_{j=0}^{t-1}\left(\begin{array}{c}
C t \\
j
\end{array}\right)\left(\frac{1}{2}\right)^{C t-j} \leq\left(\begin{array}{c}
C t \\
t
\end{array}\right) \frac{t}{2^{(C-1) t}} \\
& \leq\left(\frac{(5 / 4)^{C-1} C^{C}}{2^{C-1}(C-1)^{C-1}}\right)^{t} \leq \kappa(C)^{t}
\end{aligned}
$$

with $\kappa(C) \equiv 2 C e(2 / 3)^{C} \rightarrow 0$ as $C \rightarrow \infty$. We used here the fact that fewer than $t$ of the differences $\tau_{j}-\tau_{j-1}$ can exceed 1 in the second inequality, and Stirling's formula in the fourth.

Let now $T$ be the solution of (1.1) with $A=A_{n}$ and $T_{0} \equiv \chi_{\mathbb{R}^{-} \times \mathbb{T}^{d-1}}$. If $\phi$ solves (2.2) with $A=A_{n}$ and $\phi_{0} \equiv T_{0}$, then we have by (2.5) for $x(s) \equiv(s, 0, \ldots, 0)$,

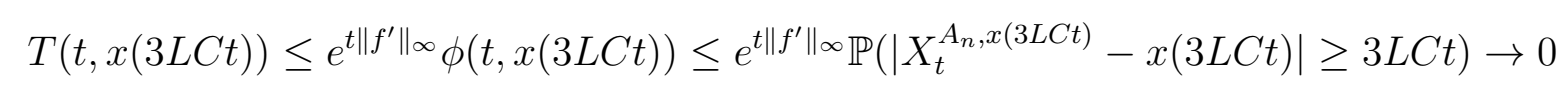

as $t \rightarrow \infty$, provided $C$ is large enough. On the other hand, it is well known that $T(t, x(c t)) \rightarrow$ 1 as $t \rightarrow \infty$ when $c<c_{*}\left(A_{n}\right)$ [4, 20, 21]. This means $c_{*}\left(A_{n}\right) \leq 3 L C$ and we are done.

Lemma 3.2. Consider the setting of Theorem 1.1 with $D=\mathbb{R} \times \mathbb{T}^{d-1}$, and let $A_{n} \rightarrow \infty$ be such that Lemma 2.2(ii) holds. Then there is compactly supported $T_{0}(x) \in[0,1]$ such that the solution $T$ of (1.1) with $A=A_{n}$ does not quench for any $n$. 
Proof. By comparison theorems, we only need to consider $f$ of ignition type - with $\theta_{0}>0$. We again choose $L \in \mathbb{N}$ that satisfies Lemma 2.2(ii) for $t=1$ and $\varepsilon=\frac{1}{2}$. We next note that there is $\delta>0$ such that

$$
\mathbb{P}\left(\left|X_{t}^{A_{n}, x}-x\right| \geq t^{8 / 15}\right) \leq e^{-t^{\delta}}
$$

for all large enough $t$ and all $x \in D$ and $n$. Indeed, assume $x_{1} \in \mathbb{Z}$ and $t \in \mathbb{Z}$ (the general case follows immediately from this), and let $j(t)=\inf \left\{j \mid \tau_{j}>t\right\}$, with $\tau_{j}$ from the proof of Lemma 3.1. Then that proof shows that for $C \in \mathbb{Z}$ we have

$$
\mathbb{P}(j(t)>C t)=\mathbb{P}\left(\tau_{C t} \leq t\right) \leq \kappa(C)^{t}
$$

with $\kappa(C)<1$ if $C$ is large. On the other hand, symmetry of $u$ across each hyperplane $x=k \in \mathbb{Z}$ shows that $Y_{j} \equiv\left(X_{\tau_{j}}^{A_{n}, x}-X_{\tau_{j-1}}^{A_{n}, x}\right)_{1}$ are iids with $\mathbb{P}\left(Y_{j}= \pm L\right)=\frac{1}{2}$. This gives

$$
\mathbb{P}\left(\left|X_{j(t)}^{A_{n}, x}-x\right| \geq L(C t)^{9 / 17} \mid j(t) \leq C t\right) \leq e^{-(C t)^{\delta}}
$$

for some $\delta>0$ by

$$
\begin{aligned}
\sum_{k=0}^{\frac{1}{2}\left(j-j^{\delta+\frac{1}{2}}\right)} \frac{\left(\begin{array}{l}
j \\
k
\end{array}\right)}{2^{j}} & \approx\left(1+j^{\delta-\frac{1}{2}}\right)^{-\frac{1}{2}\left(j+j^{\delta+\frac{1}{2}}\right)}\left(1-j^{\delta-\frac{1}{2}}\right)^{-\frac{1}{2}\left(j-j^{\delta+\frac{1}{2}}\right)} \\
& =\left[\left(1-j^{2 \delta-1}\right)^{-j^{1-2 \delta}}\left(1+j^{\delta-\frac{1}{2}}\right)^{-j^{\frac{1}{2}-\delta}}\left(1-j^{\delta-\frac{1}{2}}\right)^{j^{\frac{1}{2}-\delta}}\right]^{j^{2 \delta} / 2} \approx e^{-j^{2 \delta} / 2},
\end{aligned}
$$

where we used Stirling's formula again. This, the fact that $\left|X_{\tau_{j(t)}}^{A_{n}, x}-X_{t}^{A_{n}, x}\right| \leq L$ (by the definition of $\tau_{j}$ and $j(t)$ ), and (3.2) yield (3.1) for large enough $t$ (with a different $\delta>0$ ).

We will also need the conclusion of Lemma 3.1 in [9] which says that there is $\tilde{c}>0$ such that for any $x \in D, m \in \mathbb{Z}, A \in \mathbb{R}$, incompressible $u$, and $t \geq 1$ we have

$$
\mathbb{P}\left(\left(X_{t}^{A, x}\right)_{1} \in[m, m+1]\right) \leq \tilde{c} t^{-1 / 2} .
$$

We note that [9] only considers $d=2$, but the general case is identical.

Let us now take non-negative $\psi_{0} \in C(\mathbb{R}) \cap C^{3}([-2,2])$ such that

$$
\begin{gathered}
\operatorname{supp} \psi_{0}=[-2,2], \\
\psi_{0}(s)=\psi_{0}(-s) \quad \text { and } \quad \psi_{0}(0)=\frac{2+\theta_{0}}{3}, \\
\psi_{0}(s)=\frac{1+\theta_{0}}{6}\left[(3-|s|)^{2}-1\right] \text { for }|s| \in[1,2], \\
\psi_{0}^{\prime} \text { is decreasing on }[-1,1] .
\end{gathered}
$$

Note that this means that $\psi_{0}$ is non-negative, symmetric, non-increasing on $\mathbb{R}^{+}$, and convex where $f\left(\psi_{0}(s)\right)=0$. We then let

$$
T_{0}(x) \equiv \psi_{0}\left(\frac{x_{1}}{M}\right) \geq 0
$$

with a large $M \in \mathbb{Z}$ to be determined later. We will show using the properties of $\psi_{0}$ that if $T$ solves (1.1) with $A=A_{n}$, then for $\tau \equiv M^{3 / 2}$ we have

$$
T(\tau, x) \geq T_{0}(x)
$$

(which gives the desired result by comparison theorems). 
Let $\varepsilon$ be such that $\psi_{0}(1+\varepsilon)=\frac{1+2 \theta_{0}}{3}$ and $M$ such that $\varepsilon M+M^{4 / 5} \leq M-2$. Let $\phi$ be the solution of (2.2) with $\phi_{0} \equiv T_{0}$ and assume first that $x_{1} \in\left[(1+\varepsilon) M, 2 M-M^{4 / 5}\right] \cap \mathbb{Z}$. Let $x^{\prime} \equiv\left(x_{2}, \ldots, x_{d}\right)$. Then by (2.3) $)$, monotonicity of $\psi_{0}$ on $\mathbb{R}^{+}$, and symmetry of $u$,

$$
\begin{aligned}
& \phi(\tau, x) \geq \sum_{m=-M^{4 / 5}-1}^{M^{4 / 5}} \mathbb{P}\left(\left(X_{\tau}^{A_{n}, x}\right)_{1} \in\left[x_{1}+m, x_{1}+m+1\right]\right) \phi_{0}\left(x_{1}+m+1, x^{\prime}\right) \\
& \quad=\sum_{m=0}^{M^{4 / 5}} \mathbb{P}\left(\left(X_{\tau}^{A_{n}, x}\right)_{1} \in\left[x_{1}+m, x_{1}+m+1\right]\right)\left(\phi_{0}\left(x_{1}+m+1, x^{\prime}\right)+\phi_{0}\left(x_{1}-m, x^{\prime}\right)\right) .
\end{aligned}
$$

We have

$\psi_{0}\left(\frac{x_{1}+m+1}{M}\right)+\psi_{0}\left(\frac{x_{1}-m}{M}\right)=2 \psi_{0}\left(\frac{x_{1}+\frac{1}{2}}{M}\right)+\psi_{0}^{\prime \prime}\left(\frac{x_{1}+\frac{1}{2}}{M}\right)\left(\frac{m+\frac{1}{2}}{M}\right)^{2}+O\left(\left(\frac{m}{M}\right)^{3}\right)$,

and $\tau=M^{3 / 2}$ together with (3.1) implies that the sum of the $\mathbb{P}(\cdot)$ terms in (3.5) is larger than $\frac{1}{2}\left(1-e^{-\tau^{\delta}}\right)=\frac{1}{2}\left(1-e^{-M^{3 \delta / 2}}\right)$. This and $\psi_{0}^{\prime \prime}(s)=\frac{1+\theta_{0}}{3}$ for $s \in(1,2)$ yields

$$
\phi(\tau, x) \geq\left(1-e^{-M^{3 \delta / 2}}\right) \phi_{0}\left(x_{1}+\frac{1}{2}, x^{\prime}\right)+\frac{1+\theta_{0}}{12}\left(4 \tilde{c} M^{1 / 4}\right)^{-2}+O\left(M^{-3 / 5}\right),
$$

where we also used that (3.3) gives

$$
\mathbb{P}\left(\left|X_{\tau}^{A_{n}, x}-x\right| \geq \frac{M^{3 / 4}}{4 \tilde{c}}\right) \geq \frac{1}{2}
$$

Since $\phi_{0}(x)-\phi_{0}\left(x_{1}+\frac{1}{2}, x^{\prime}\right)=O\left(M^{-1}\right)$, this means

$$
\phi(\tau, x) \geq \phi_{0}(x)+c^{\prime} M^{-1 / 2}
$$

for some $c^{\prime}>0$ and any large enough $M$.

The same argument applies for any $\tau^{\prime} \in[\tau / 2, \tau]$ (with a uniform $c^{\prime}$ ) in place of $\tau$. This, Lemma 2.3, and the fact that $\phi_{0}$ varies on a scale $O\left(M^{-1}\right)$ on $[\lfloor x\rfloor,\lfloor x\rfloor+1] \times \mathbb{T}^{d-1}$ yield (3.6)) for any $x_{1} \in\left[(1+\varepsilon) M, 2 M-M^{4 / 5}\right]$, provided $M$ is large enough. If $x_{1} \in\left[2 M-M^{4 / 5}, 2 M\right]$, then (3.6) follows in the same way because $\psi_{0}(s)>\frac{1+\theta_{0}}{6}\left[(3-|s|)^{2}-1\right]$ for $s \in(2,3)$. And if $x_{1}>2 M$, then (3.6) is immediate from $\phi(\tau, x) \geq 0$.

Symmetry and $T \geq \phi$ give (3.4) whenever $|x| \geq(1+\varepsilon) M$, so let us now consider $|x| \leq$ $(1+\varepsilon) M$. As above we obtain for large $M$,

$$
\phi(\tau, x) \geq \phi_{0}(x)-c^{\prime} M^{-1 / 2},
$$

where $c^{\prime}$ only depends on $\left\|\psi_{0}^{\prime \prime}\right\|_{\infty}$. We now choose a convex $g: \mathbb{R}^{+} \rightarrow \mathbb{R}^{+}$with $g(s) \leq f(s)$ for $s \leq \frac{3+\theta_{0}}{4}$ and $g(s) \geq \alpha$ for some $\alpha>0$ and all $s \geq \frac{1+3 \theta_{0}}{4}$. Define $\beta>0$ so that if $\gamma(0)=\frac{2+\theta_{0}}{3}$ and $\gamma^{\prime}(s)=g(\gamma(s))$, then $\gamma(\beta)=\frac{3+\theta_{0}}{4}$. Next let $\tilde{f} \equiv \frac{\beta}{\tau} g \leq g$ when $\tau=M^{3 / 2} \geq \beta$ and let $w:\left(\mathbb{R}^{+}\right)^{2} \rightarrow \mathbb{R}^{+}$satisfy $w(0, s)=s$ and

$$
w_{t}(t, s)=\tilde{f}(w(t, s)) .
$$


Notice that

$$
w\left(\tau, \frac{2+\theta_{0}}{3}\right)=\frac{3+\theta_{0}}{4} \quad \text { and } \quad w(\tau, s) \geq s+\alpha \beta \text { for } s \geq \frac{1+3 \theta_{0}}{4} .
$$

It is easy to show using $\tilde{f}^{\prime}, \tilde{f}^{\prime \prime} \geq 0$ that $w_{s}, w_{s s} \geq 0$. It then follows that $\tilde{T}(t, x) \equiv w(t, \phi(t, x))$ is a sub-solution of (1.1) with $A=A_{n}$ and $\tilde{T}_{0}=T_{0}$ as long as $\|\tilde{T}(t, \cdot)\|_{\infty} \leq \frac{3+\theta_{0}}{4}$ (so that $\tilde{f}(\tilde{T}) \leq f(\tilde{T}))$. Since $\|\phi\|_{\infty} \leq \psi_{0}(0)=\frac{2+\theta_{0}}{3}$, this is true for all $t \leq \tau$ by (3.8) and $w_{t}, w_{s} \geq 0$. But then $T(\tau, x) \geq \tilde{T}(\tau, x)$, while large enough $M$ guarantees for $|x| \leq(1+\varepsilon) M$,

$$
\phi(\tau, x) \geq \phi_{0}(x)-c^{\prime} M^{-1 / 2} \geq \frac{1+2 \theta_{0}}{3}-c^{\prime} M^{-1 / 2} \geq \frac{1+3 \theta_{0}}{4} .
$$

So for these $x$ by (3.8),

$$
T(\tau, x) \geq \tilde{T}(\tau, x) \geq \phi(\tau, x)+\alpha \beta \geq \phi_{0}(x)-c^{\prime} M^{-1 / 2}+\alpha \beta \geq \phi_{0}(x)=T_{0}(x)
$$

when $M$ is large. This is (3.4) and thus concludes the proof.

\section{Proof of Theorem 1.1: Part II}

We now assume that $u$ and $f$ are as in Theorem 1.1 and $A_{n} \rightarrow \infty$ is such that Lemma 2.2(i) holds. We will then show that $\limsup _{n \rightarrow \infty} c_{*}\left(A_{n}\right)=\infty$, and that there is $c>0$ such that if $f$ is of ignition type with $\|f(s) / s\|_{\infty} \leq c$, then any compactly supported initial datum $T_{0}$ for (1.1) is quenched by some flow $A_{n} u$. The analysis in this section applies in two dimensions only, so we will consider $d=2$ and $D=\mathbb{R} \times \mathbb{T}$.

Lemma 4.1. Consider the setting of Theorem 1.1 with $D=\mathbb{R} \times \mathbb{T}$ and let $A_{n} \rightarrow \infty$ be such that Lemma 2.2(i) holds. Then $\lim \sup _{n \rightarrow \infty} c_{*}\left(A_{n}\right)=\infty$.

Proof. Assume that $c_{*}\left(A_{n}\right) \leq c_{0}<\infty$ for all $n$ and let $T$ be a pulsating front solution of (1.1) with $A=A_{n}$ and speed $c_{*}\left(A_{n}\right)$, that is,

$$
\begin{gathered}
T\left(t+c_{*}\left(A_{n}\right)^{-1}, x_{1}+1, x_{2}\right)=T\left(t, x_{1}, x_{2}\right), \\
T\left(t, \pm \infty, x_{2}\right)=\frac{1}{2} \mp \frac{1}{2} \quad \text { uniformly in } x_{2}
\end{gathered}
$$

(recall that $u$ has period 1 in $x_{1}$ ). We note that [2] shows

$$
T_{t}(t, x) \geq 0 \text {. }
$$

Integrating (1.1) over $\left[0, c_{*}\left(A_{n}\right)^{-1}\right] \times D$ and using (4.1) and incompressibility of $u$, we obtain

$$
1=\int_{0}^{c_{*}\left(A_{n}\right)^{-1}} \int_{D} f(T(t, x)) d x d t
$$

Next we multiply (1.1) by $T$ and again integrate as above to get

$$
\frac{1}{2}=\int_{0}^{c_{*}\left(A_{n}\right)^{-1}} \int_{D} T(t, x) f(T(t, x))-|\nabla T(t, x)|^{2} d x d t \leq 1-\int_{0}^{c_{*}\left(A_{n}\right)^{-1}} \int_{D}|\nabla T(t, x)|^{2} d x d t .
$$

This means that for some $t \in\left[0, c_{*}\left(A_{n}\right)^{-1}\right]$ (which we take to be 0 by translating $T$ in time),

$$
\int_{D} f(T(0, x)) d x \leq 2 c_{0}
$$




$$
\int_{D}|\nabla T(0, x)|^{2} d x \leq c_{0}
$$

We will now show that (4.1)-(4.4) force the reaction zone (front width) to be bounded in the following sense. Let $D_{\varepsilon}^{-}$be the rightmost cell $\left[m_{\varepsilon}^{-}, m_{\varepsilon}^{-}+1\right] \times \mathbb{T}$ such that inf ${ }_{x \in D_{\varepsilon}^{-}} T(0, x) \geq 1-\varepsilon$ (i.e., $m_{\varepsilon}^{-}$is the largest integer for which this condition holds). We also let $D_{\varepsilon}^{+}$be the leftmost cell $\left[m_{\varepsilon}^{+}, m_{\varepsilon}^{+}+1\right] \times \mathbb{T}$ such that $\sup _{x \in D_{\varepsilon}^{+}} T(0, x) \leq 1-\varepsilon$. Obviously $m_{\varepsilon}^{-}<m_{\varepsilon}^{+}$. We will now show that for each small $\varepsilon>0$ there is $L_{\varepsilon}<\infty$ such that for each $n$ we have

$$
m_{10 \varepsilon}^{+}-m_{\varepsilon}^{-} \leq L_{\varepsilon} \text {. }
$$

Assume for a moment that (4.5) holds. Periodicity and (2.8) tell us that there are $n$ and $x \in D_{\varepsilon}^{-}$such that

$$
\mathbb{P}\left(\left|X_{\tau}^{A_{n}, x}-x\right| \geq L_{\varepsilon}\right)>\frac{1}{2}
$$

for $\tau \equiv \varepsilon\left\|f^{\prime}\right\|_{\infty}^{-1}>0$. Since $x_{1} \geq m_{\varepsilon}^{-} \geq m_{10 \varepsilon}^{+}-L_{\varepsilon}$, symmetry of $u$ implies

$$
\mathbb{P}\left(\left(X_{\tau}^{A_{n}, x}\right)_{1} \geq m_{10 \varepsilon}^{+}\right)>\frac{1}{4} \text {. }
$$

Using (2.5) and (2.3) we have

$$
T(\tau, x) \leq e^{\tau\left\|f^{\prime}\right\|_{\infty}}\left(\frac{3}{4}+\frac{1-10 \varepsilon}{4}\right)<1-\varepsilon \leq T(0, x)
$$

if $\varepsilon>0$ is small. This contradicts (4.2), so our assumption $c_{*}\left(A_{n}\right) \leq c_{0}<\infty$ must be invalid. Thus the proof will be finished if we establish (4.5) for all small $\varepsilon>0$.

Let us consider an arbitrary small $\varepsilon>0$ such that $f$ is bounded away from zero on $\left[1-13 \varepsilon, 1-\frac{\varepsilon}{3}\right]$ and assume, towards contradiction, that for each $L \in \mathbb{N}$ there is $n$ such that

$$
m_{10 \varepsilon}^{+}-m_{\varepsilon}^{-} \geq 10 L \text {. }
$$

Let $T_{0}(x) \equiv T(0, x)$,

$$
\bar{T}_{0}(x) \equiv \int_{\left[\left\lfloor x_{1}\right\rfloor,\left\lfloor x_{1}\right\rfloor+1\right] \times \mathbb{T}} T_{0}(x) d x,
$$

and denote $D_{j} \equiv\left[m_{\varepsilon}^{-}+j, m_{\varepsilon}^{-}+j+1\right] \times \mathbb{T}$. Then (4.4) and Poincaré inequality (with constant $C$ ) imply that for each small $\delta>0$ and $L \equiv\left\lceil C c_{0} / \delta\right\rceil$, at least $7 L$ of the cells $D_{j}$, $j=L, \ldots, 9 L$, satisfy

$$
\left\|T_{0}-\bar{T}_{0}\right\|_{L^{2}\left(D_{j}\right)}^{2} \leq C\left\|\nabla T_{0}\right\|_{L^{2}\left(D_{j}\right)}^{2} \leq \delta
$$

Hence there are at least $\left\lfloor\frac{3 L}{5}\right\rfloor$ disjoint 5 -tuples of consecutive cells satisfying (4.7). Then (4.3), $f$ bounded away from zero on $\left[1-13 \varepsilon, 1-\frac{\varepsilon}{3}\right]$, and $\bar{T}_{0}\left(D_{j}\right)$ decreasing in $j$ (by (4.2)) imply that for some $j_{0} \in[L, 9 L]$ we must have either (4.7) and $\bar{T}_{0}\left(D_{j}\right) \leq 1-12 \varepsilon$ for $j=j_{0}-2, \ldots, j_{0}+2$, or (4.7) and $\bar{T}_{0}\left(D_{j}\right) \geq 1-\frac{\varepsilon}{2}$ for $j=j_{0}-2, \ldots, j_{0}+2$ (provided $\delta$ is small enough and $L$ large).

Let us assume the case $\bar{T}_{0}\left(D_{j}\right) \leq 1-12 \varepsilon$ for $j=j_{0}-2, \ldots, j_{0}+2, j_{0} \in[L, 9 L]$. Then (4.2) and (4.6) say that there must be $y \in D_{j_{0}}$ such that for $t \geq 0$,

$$
T(t, y) \geq T_{0}(y) \geq 1-10 \varepsilon \text {. }
$$


Let $S_{2 \gamma}^{-} \subset D_{j_{0}-2} \cup D_{j_{0}-1} \cup D_{j_{0}}$ be the square of a small side $2 \gamma>0$ (to be chosen later) centered at $y^{-} \equiv y-(1,0)$ and denote by $\Gamma^{-}$the intersection of $S_{2 \gamma}^{-}$with the connected component $\Omega^{-}$of the set $\left\{x \mid T_{0}(x) \geq 1-11 \varepsilon\right\}$ containing $y^{-}$(recall that that $T_{0}\left(y^{-}\right) \geq T_{0}(y) \geq 1-10 \varepsilon$ ).

If $\Gamma^{-}$has diameter less than $\gamma$ (in particular, $\Gamma^{-}=\Omega^{-} \subseteq S_{2 \gamma}^{-}$), then for $\Gamma \equiv \Gamma^{-}+(1,0)$, all $x \in \partial \Gamma$, and all $t \leq c_{*}\left(A_{n}\right)^{-1}$,

$$
T(t, x) \leq T(0, x-(1,0)) \leq 1-11 \varepsilon
$$

by (4.1) and (4.2). It follows by comparison that $T(t, x) \leq e^{t\left\|f^{\prime}\right\|_{\infty}}(R(t, x)+1-11 \varepsilon)$ where $R(t, x)$ solves (2.2) on $S_{2 \gamma} \equiv S_{2 \gamma}^{-}+(1,0)$ with Dirichlet boundary conditions and $R(0, x)=$ $11 \varepsilon \chi_{\Gamma}(x)$. But then the uniform bound in Lemma 2.3 and parabolic scaling in $(t, x)$ gives that for any $t>0$ there is small enough $\gamma>0$ such that $\|R(t, x)\|_{\infty} \leq \frac{\varepsilon}{2}$, and if $t$ is chosen small enough (and $\gamma$ accordingly), then $T(t, y)<1-10 \varepsilon$ follows. This clearly contradicts (4.8).

If instead (for the chosen $\gamma$ ) the set $\Gamma^{-} \subset D_{j_{0}-2} \cup D_{j_{0}-1} \cup D_{j_{0}}$ has diameter at least $\gamma$, then $\bar{T}_{0}\left(D_{j}\right) \leq 1-12 \varepsilon$ and $\inf T_{0}\left(\Gamma^{-}\right) \geq 1-11 \varepsilon$ imply that the second inequality in (4.7) must be violated for at least one of $j=j_{0}-2, j_{0}-1, j_{0}$, provided $\delta>0$ is chosen small enough (depending on $\gamma, \varepsilon$ ). Indeed - if $\left\|\nabla T_{0}\right\|_{L^{2}\left(D_{j}\right)}^{2}$ is small enough, then $T$ must be close to $1-11 \varepsilon$ on some vertical line passing through $\Gamma^{-}$, and then $T$ must be close to $1-11 \varepsilon$ on most horizontal lines inside $D_{j}$ by the same argument. This contradicts $\bar{T}_{0}\left(D_{j}\right) \leq 1-12 \varepsilon$.

Finally, if we instead assume $\bar{T}_{0}\left(D_{j}\right) \geq 1-\frac{\varepsilon}{2}$ for $j=j_{0}-2, \ldots, j_{0}+2$ and $T(t, y) \leq$ $T_{0}(y-(1,0)) \leq 1-\varepsilon$ for small $t \geq 0$, a similar argument again leads to contradiction. This means that (4.6) cannot hold for small $\varepsilon>0$ and (4.5) follows. The proof is finished.

Lemma 4.2. Consider the setting of Theorem 1.1 with $D=\mathbb{R} \times \mathbb{T}$. There is $c>0$ such that if $f$ is of ignition type with $\|f(s) / s\|_{\infty} \leq c$ and $A_{n} \rightarrow \infty$ is such that Lemma 2.2(i) holds, then for any compactly supported $T_{0}(x) \in[0,1]$ there is $n$ such that the solution $T$ of (1.1) with $A=A_{n}$ quenches.

Remark. We note that $c$ is from Lemma 2.3 and can be easily evaluated from its proof.

Proof. By comparison theorems, it is sufficient to consider initial data $T_{0}(x) \equiv \chi_{[-L, L]}\left(x_{1}\right)$ for all $L \in \mathbb{N}$. Let $\phi$ be the solution of (2.2) with $A=A_{n}$ and initial datum $\phi_{0} \equiv T_{0}$. We first claim that for each $\tau, \delta>0$ there is $n$ and a continuous curve $h:[0,1] \rightarrow[0,1] \times \mathbb{T}$ such that $(h(0))_{1}=0$ and $(h(1))_{1}=1$, and for all $s \in[0,1]$ and $t \geq \tau$,

$$
\phi(t, h(s)) \leq \delta
$$

To this end we let $\psi$ be the solution of (2.2) with initial condition $\psi_{0} \equiv \chi_{[-K-2, K]}\left(x_{1}\right)$ where $K \geq 3 L \delta^{-1}$. By periodicity of $u$ and (2.8), there must be $n$ (which will be kept constant from now on) and $y \in[-1,0] \times \mathbb{T}$ such that

$$
\psi(\tau, y)=\mathbb{P}\left(\left(X_{\tau}^{A_{n}, y}\right)_{1} \in[-K-2, K]\right) \leq \frac{\delta}{2} .
$$

The maximum principle for (2.2) implies that the connected component of the set

$$
\left\{(t, x) \in[0, \tau] \times D \mid \psi(t, x) \leq \frac{\delta}{2}\right\}
$$


containing $(\tau, y)$ must intersect

$$
\left\{x \in D \mid \psi(0, x) \leq \frac{\delta}{2}\right\}=(\mathbb{R} \backslash[-K-2, K]) \times \mathbb{T} .
$$

Since by symmetry $\psi\left(t, x_{1}, x_{2}\right)=\psi\left(t,-2-x_{1}, x_{2}\right)$ for $x_{1} \geq 0$, this means that there is a curve $h(s)$ joining $\{0\} \times \mathbb{T}$ and $\{K\} \times \mathbb{T}$ such that for each $s$ there is $\tau_{s} \leq \tau$ with

$$
\psi\left(\tau_{s}, h(s)\right)=\mathbb{P}\left(\left(X_{\tau_{s}}^{A_{n}, h(s)}\right)_{1} \in[-K-2, K]\right) \leq \frac{\delta}{2} .
$$

Lemma 2.1(iii) and the definition of $K$ then mean that for all $t \geq \tau$,

$$
\left.\phi(t, h(s))=\mathbb{P}\left(\left|X_{t}^{A_{n}, h(s)}\right| \leq L\right]\right) \leq \frac{\delta}{2}+\left(1-\frac{\delta}{2}\right) \frac{\delta}{3} \leq \delta
$$

which is (4.9) (after reparametrization of $h$ and restriction to $s \in[0,1]$ ).

Symmetry of $u$ and $\phi_{0}$ implies that (4.9) holds for $h(s)$ extended to $s \in[-1,1]$ by $h(-s)=$ $\left(-(h(s))_{1},(h(s))_{2}\right)$. Finally, (4.9) applies to $h(s)$ extended periodically (with period 2) onto $\mathbb{R}$. This last claim holds because $\phi(t, x) \geq \phi(t, x+(2,0))$ when $x_{1} \geq-1$ (and $\phi(t, x) \geq$ $\phi(t, x-(2,0))$ when $\left.x_{1} \leq 1\right)$, which in turn follows because $\phi(t, x)-\phi(t, x+(2,0))$ solves (2.2) with initial datum that is symmetric across $x_{1}=-1$ and non-negative on $[-1, \infty) \times \mathbb{T}$ (and hence stays such by the symmetry of $u$ ).

This means that $\|\phi(t+\tau, \cdot)\|_{\infty} \leq\|\psi(t, \cdot)\|_{\infty}+\delta$ where $\psi$ is the solution of (2.2) on $2 \mathbb{T} \times \mathbb{T}$ with $\psi_{0} \equiv 1$ and $\psi(t, h(s))=0$ for all $t>0$ and $s \in[0,2]$. Since the Poincaré inequality and the proof of Lemma 2.3 extend to this setting with the same universal constant $c>0$, we obtain that $\|\phi(t, \cdot)\|_{\infty} \leq \delta+2 e^{-c(t-\tau)}$. If now $\|f(s) / s\|_{\infty}=c^{\prime}\langle c$ and $\tau, \delta>0$ are chosen small enough depending on $c-c^{\prime}$ (and $n$ accordingly), we obtain $\left\|T\left(t_{0}, \cdot\right)\right\|_{\infty} \leq$ $e^{c^{\prime} t_{0}}\left(\delta+2 e^{c \tau} e^{-c t_{0}}\right) \leq \theta_{0}$ for some $t_{0}$. The maximum principle then implies $\|T(t, \cdot)\|_{\infty} \leq \theta_{0}$ for any $t \geq t_{0}$ and quenching follows.

The proof of Theorem [1.1 is now based on the last four lemmas and this result from [18]:

Lemma 4.3. Assume the setting of Theorem 1.1 with $f$ a KPP nonlinearity and $D=\mathbb{R} \times \mathbb{T}$.

(i) If $(1.2)$ on $2 \mathbb{T} \times \mathbb{T}$ has a solution $\psi \in H^{1}(2 \mathbb{T} \times \mathbb{T})$, then $(1.3)$ holds.

(ii) If (1.2) has no $H^{1}(2 \mathbb{T} \times \mathbb{T})$-solutions, then (1.4) holds.

Proof of Theorem 1.1. If (1.2) has a solution $\psi \in H^{1}(2 \mathbb{T} \times \mathbb{T})$, then $c_{*}\left(A_{n}\right)$ is bounded for any KPP $f$ and any $A_{n} \rightarrow \infty$, and so Lemma 4.1 gives Lemma 2.2(ii). Lemmas 3.1 and 3.2 now give (i) for any $f$. Note that if each sequence $A_{n}$ does not quench some compactly supported initial datum $T_{0}$ for (1.1) with $A=A_{n}$, then there is $T_{0}$ that is not quenched by any $A$. This holds because if each $T_{0}(x) \equiv \chi_{[-n, n]}\left(x_{1}\right)$ is quenched by some $A_{n}$, then this sequence would yield a contradiction.

If, on the other hand, (1.2) has no $H^{1}(2 \mathbb{T} \times \mathbb{T})$-solutions, then $c_{*}\left(A_{n}\right) \rightarrow \infty$ for any $\operatorname{KPP} f$ and any $A_{n} \rightarrow \infty$, and so Lemma 3.1 gives Lemma 2.2(i). Lemma 4.1 now gives (1.4) for any $f$. The claim about the existence of $l_{0}$ follows from the fact that $T$ solves $T_{t}-A u^{(l)} \cdot \nabla T=$ $\Delta T+f(T)$ on $\mathbb{R} \times l \mathbb{T}$ if and only if $S(t, x) \equiv T\left(l^{2} t, l x\right)$ solves $S_{t}-A l u \cdot \nabla S=\Delta S+l^{2} f(S)$ on $\mathbb{R} \times \mathbb{T}$. Comparison theorems and $f \geq 0$ then show that if $u^{(l)}$ is quenching for $f$, then so is $u^{(\tilde{l})}$ for any $\tilde{l}<l$. This only guarantees $l_{0} \in[0, \infty]$, but $l_{0}<\infty$ follows from Theorem 
8.2 in [23] and the fact that the flow $u$ leaves the bounded domain $[0, p] \times \mathbb{T}$ invariant. For ignition reactions Lemma 4.2 shows $l_{0}>0$ - if each $T_{0}$ is quenched by at least one $A_{n} u$ for any sequence $A_{n} \rightarrow \infty$, then each $T_{0}$ is quenched by $A u$ for all large $A$.

Finally, we provide the following extension of Theorem 1.1(ii) to some positive reactions.

Corollary 4.4. The claim $l_{0}>0$ in Theorem 1.1(ii) holds for any combustion-type reaction satisfying $f(s) \leq \alpha s^{\beta}$ for some $\alpha>0, \beta>3$, and all $s \in[0,1]$.

Proof. By the proof of Theorem 1.1, it is sufficient to show that there is $l>0$ such that $u$ is quenching for $l^{2} f(s)$. The proof is essentially identical to that of Theorem 8.3 in [23]. We let $I_{A} \equiv \int_{0}^{\infty}\|\phi(t, \cdot)\|_{\infty}^{\beta-1} d t$ where $\phi$ is the solution of (2.2) and $\phi_{0}(x) \equiv T_{0}(x)$. It follows from [14] (see also [22, Lemma 2.1]) that $u$ is quenching for $l^{2} f(s)$ when for each compactly supported $T_{0}$ there is $A_{0}$ such that $l^{2} \alpha(\beta-1) I_{A}<1$ whenever $A \geq A_{0}$. So fix $T_{0}$ and notice that the bound $\|\phi(t, \cdot)\|_{\infty} \leq \tilde{c}\left|\operatorname{supp} T_{0}\right| t^{-1 / 2}$ for $t \geq 1$, which follows from (3.3), gives $\int_{t_{0}}^{\infty}\|\phi(t, \cdot)\|_{\infty}^{\beta-1} d t \leq 1$ if $t_{0}$ is chosen appropriately (depending on $\tilde{c}\left|\operatorname{supp} T_{0}\right|$ ). For $t \leq t_{0}$ we use the bound $\|\phi(t, \cdot)\|_{\infty} \leq 5 e^{-c t}$, which follows from the proof of Lemma 4.2 (with the same c) provided $A_{0}$ is chosen large enough so that $\delta$ in that proof is smaller than $e^{-c t_{0}}$ for each $A \geq A_{0}$ (and $\tau$ is such that $e^{c \tau} \leq 2$ ). This choice is possible because each sequence $A_{n} \rightarrow \infty$ has a term $A_{n}$ guaranteeing $\delta<e^{-c t_{0}}$. Hence for $A \geq A_{0}$ we have

$$
\int_{0}^{t_{0}}\|\phi(t, \cdot)\|_{\infty}^{\beta-1} d t \leq \int_{0}^{\infty}\left(5 e^{-c t}\right)^{\beta-1} d t \equiv C<\infty .
$$

Now let $l>0$ be such that $l^{2} \alpha(\beta-1)(1+C)<1$, and we are done.

\section{REFERENCES}

[1] B. Audoly, H. Berestycki and Y. Pomeau, Réaction diffusion en écoulement stationnaire rapide, C. R. Acad. Sci. Paris 328, Série IIb (2000), 255-262.

[2] H. Berestycki and F. Hamel, Front propagation in periodic excitable media, Comm. Pure and Appl. Math. 55 (2002), 949-1032.

[3] H. Berestycki, The influence of advection on the propagation of fronts in reaction-diffusion equations, Nonlinear PDEs in Condensed Matter and Reactive Flows, NATO Science Series C, 569, H. Berestycki and Y. Pomeau eds, Kluwer, Doordrecht, 2003.

[4] H. Berestycki, F. Hamel and N. Nadirashvili, The speed of propagation for KPP type problems, I Periodic framework, J. European Math. Soc. 7 (2005), 173-213.

[5] H. Berestycki, F. Hamel and N. Nadirashvili, Elliptic eigenvalue problems with large drift and applications to nonlinear propagation phenomena, Comm. Math. Phys. 253 (2005), 451-480.

[6] P. Constantin, A. Kiselev, A. Oberman and L. Ryzhik, Bulk burning rate in passive-reactive diffusion, Arch. Ration. Mech. Anal. 154 (2000), 53-91.

[7] P. Constantin, A. Kiselev, L. Ryzhik, Quenching of flames by fluid advection, Comm. Pure Appl. Math. 54 (2001), 1320-1342.

[8] P. Constantin, A. Kiselev, L. Ryzhik, and A. Zlatoš, Diffusion and Mixing in Fluid Flow, Ann. of Math. (2), to appear.

[9] A. Fannjiang, A. Kiselev and L. Ryzhik, Quenching of reaction by cellular flows, Geom. Funct. Anal. 16 (2006), 40-69.

[10] S. Heinze, Large convection limits for KPP fronts, Max Planck Institute for Mathematics Preprint Nr. $21 / 2005,2005$. 
[11] A. Kiselev and L. Ryzhik, Enhancement of the traveling front speeds in reaction-diffusion equations with advection, Ann. Inst. H. Poincaré Anal. Non Linéaire 18 (2001), 309-358.

[12] A. Kiselev and A. Zlatoš, Quenching of combustion by shear flows, Duke Math. J. 132 (2006), 49-72.

[13] A.N. Kolmogorov, I.G. Petrovskii and N.S. Piskunov, Étude de l'équation de la chaleur de matière et son application à un problème biologique, Bull. Moskov. Gos. Univ. Mat. Mekh. 1 (1937), 1-25.

[14] P. Meier, On the critical exponent for reaction-diffusion equations, Arch. Rational Mech. Anal. 109 (1990), 63-71.

[15] A. Novikov and L. Ryzhik, Bounds on the speed of propagation of the KPP fronts in a cellular flow, Arch. Rat. Mech. Anal. 184 (2007), 23-48.

[16] B. Øksendal, Stochastic Differential Equations, Springer-Verlag, Berlin, 1995.

[17] J.-M. Roquejoffre, Eventual monotonicity and convergence to travelling fronts for the solutions of parabolic equations in cylinders, Ann. Inst. H. Poincaré Anal. Non Linéaire 14 (1997), 499-552.

[18] L. Ryzhik and A. Zlatoš, KPP pulsating front speed-up by flows, Commun. Math. Sci. 5 (2007), 575-593.

[19] J. Smoller, Shock Waves and Reaction-Diffusion Equations, Springer-Verlag, New York, 1994.

[20] H. Weinberger, On spreading speeds and traveling waves for growth and migration models in a periodic habitat, Jour. Math. Biol. 45 (2002), 511-548.

[21] J. Xin, Existence and nonexistence of travelling waves and reaction-diffusion front propagation in periodic media, J. Stat. Phys. 73 (1993), 893-926.

[22] A. Zlatoš, Quenching and propagation of combustion without ignition temperature cutoff, Nonlinearity 18 (2005), 1463-1475.

[23] A. Zlatoš, Diffusion in fluid flow: Dissipation enhancement by flows in 2D, preprint.

[24] A. Zlatoš, Sharp Asymptotics for KPP Pulsating Front Speed-up and Diffusion Enhancement by Flows, preprint.

Department of Mathematics, University of Chicago, Chicago, il 60637, USA

EMAIL: zlatos@math.uchicago.edu 